\title{
Risk Governance, Assessment, and Economic Impacts
}

\author{
Paula Teves-Costa $^{1,2} \cdot$ José Manuel Mendes $^{3}$
}

Published online: 25 September 2017

(C) The Author(s) 2017. This article is an open access publication

Due to climate change, growing urbanization, and population migration the social and economic impacts of natural hazards have been increasing worldwide. The challenge for risk science, both methodologically and conceptually, is to integrate robust multi-hazard evaluation models with solid economic impact assessment to further improve existing civil protection plans, to promote risk awareness in social groups and local communities, and to develop a consolidated safety culture. This is in full alignment with the Sendai Framework for Disaster Risk Reduction 2015-2030 priorities 1 [understanding disaster risk, mainly paragraph 24(d)] and 2 [strengthening disaster risk governance to manage disaster risk, mainly paragraph $31(\mathrm{~d})]$.

This special issue results from the scientific meeting International Conference on Urban Risks, which took place in Lisbon, from 30 June to 2 July 2016, organized by the European Centre on Urban Risks (CERU). The meeting objectives were to reflect on the challenges put on research and practice by emerging risks, cascading disasters and cascading effects, and risk governance on local, regional, national, and global scales.

The editors selected the papers in order to give thematic coherence to this special issue. The main objective is to advance studies in disaster risk reduction across increasing

Paula Teves-Costa

mpcosta@fc.ul.pt

1 Instituto Dom Luiz, Faculdade de Ciências, Universidade de Lisboa, Campo Grande, 1749-016 Lisbon, Portugal

2 European Center on Urban Risks (CERU), 1000-146 Lisbon, Portugal

3 Center for Social Studies, Faculty of Economics, University of Coimbra, 3004-512 Coimbra, Portugal scales, mainly through the analysis of two dimensions: (1) risk governance and risk assessment; and (2) economic impacts and insurance evaluation.

The innovative aspect of this special issue lies in the conjunction of these two dimensions, exposing the need to further risk science through the complementary analysis of assessment models, economic impacts, and risk governance. Furthermore, some of the articles advance new methodologies for risk assessment, and contribute new perspectives on risk analysis and its contribution to risk governance. The articles are also diverse in the geographical areas studied, ranging from Colombia to European countries such as Portugal, Italy, and France.

Del Gobbo et al. show the limits of conventional seismic design as stipulated in Eurocode 8, mainly with respect to nonstructural repair costs. By using new methodological approaches, highlighting, among others, fragility groups, the authors propose a benchmark analysis that allows for evaluation of building retrofit alternatives and design options for new structures. The article has a direct relation to disaster impact reduction costs that are reflected in nonstructural economic losses due to seismic activity.

Children are one of the most affected groups in disasters. Although we witness a participatory turn in disaster risk reduction, Delicado et al. conclude from their study of risk governance in Portugal that children are mostly seen as victims and not direct participants in prevention and mitigation activities. This exclusionary pattern is mainly due to a nonparticipant culture and absence in recent years of disasters with high losses of human life. The authors, nevertheless, contend that growing awareness of the importance of participation of children in all disaster stages lays the groundwork for further risk governance changes 
relevant to building safer and more resilient communities and societies.

Carreño et al. discuss their holistic model for disaster risk assessment that integrates physical risk, socioeconomic fragility and addresses frequent lack of resilience by testing its application to the city of Manizales, Colombia. They conclude that the evaluation model is robust and is relevant for city disaster risk management planning, in large part because it precisely identifies pertinent differences in exposure and response to hazard risk at local levels. The authors conclude also that the holistic approach is central for stakeholders to improve their risk understanding and engage in better decision making related to disaster risk reduction.

Also for the city of Manizales, Colombia, Bernal et al. discuss and apply a multi-hazard and probabilistic risk assessment. The authors focus on seismic, landslide, and volcanic hazards. The results are integrated into land-use planning activities and emergency response plans, the last based on different risk scenarios.

Guillier presents the results of rating Action Programs for Flood Prevention (PAPI), the main instruments for flood policy planning in France. Incorporating expert valuation of flood risk and the use of Analytic Network and Analytic Hierarchy Processes, and arguing for the collective dimension of flood risk, Guillier highlights, in a sophisticated and evidence-based approach, the importance of prevention in flood risk assessment. The author shows the relevance of scientific and rigorous analytic models for policy and decision making in disaster risk prevention and reduction.

Also directly related to insurance policy and risk assessment, Salgado-Gálvez et al. present the results of a probabilistic seismic hazard analysis in Manizales, Colombia. By focusing on the water and sewage network and public and private buildings, the article shows the importance of local government in protecting the poorest socioeconomic strata of the population and promoting an insurance culture. The good practices resulting from the cooperation of local government and the private sector in risk assessment and insurance coverage could be used as a benchmark for similar experiences in other developing countries.

The last two articles of this special issue are centered on seismic risk and the economic impact of earthquakes. From a methodological perspective, Redweik et al. present and discuss the results of a 3-D study of seismic vulnerability in Lisbon. The authors undertake an analysis of all Lisbon's buildings to provide context and concentrate on three pilot areas for more detailed data; the results are oriented to three specific target groups with different aims: elected officials, planners, and experts; civil protection and emergency services agents; and the general public. Despite the value of color scale symbology for awareness campaigns, trainings, and emergency responses, the authors highlight the importance of representing the damage in a visually realistic way, thus allowing a better understanding of the damages produced by an earthquake.

Meroni et al. use data of the 2012 Emilia earthquake in Italy to assess economic loss in a study area characterized by moderate hazard intensity and high economic exposure. The results are relevant both for civil protection objectives and for insurance companies.

The articles in this special issue, although covering different scientific areas, constitute a coherent set that unequivocally shows the multidisciplinarity associated with the study and evaluation of the social and economic impacts of natural hazards. For effective risk assessment and risk governance it is necessary to take into account a wide range of studies in different scientific fields.

Open Access This article is distributed under the terms of the Creative Commons Attribution 4.0 International License (http://crea tivecommons.org/licenses/by/4.0/), which permits unrestricted use, distribution, and reproduction in any medium, provided you give appropriate credit to the original author(s) and the source, provide a link to the Creative Commons license, and indicate if changes were made. 